\title{
Teaching NeuroImages: Neuroimaging Findings in Inosine Triphosphate Pyrophosphohydrolase Deficiency
}

Karthik Muthusamy, MD, Suzanne Boyer, RDN, LD, Marc Patterson, MD, Jorgen Bierau, PhD, Saskia Wortmann, MD, PhD, and Eva Morava, MD, PhD

Neurology ${ }^{\circledR}$ 2021;97:e109-e110. doi:10.1212/WNL.0000000000011719

Figure MRI Brain at Age 4 Months

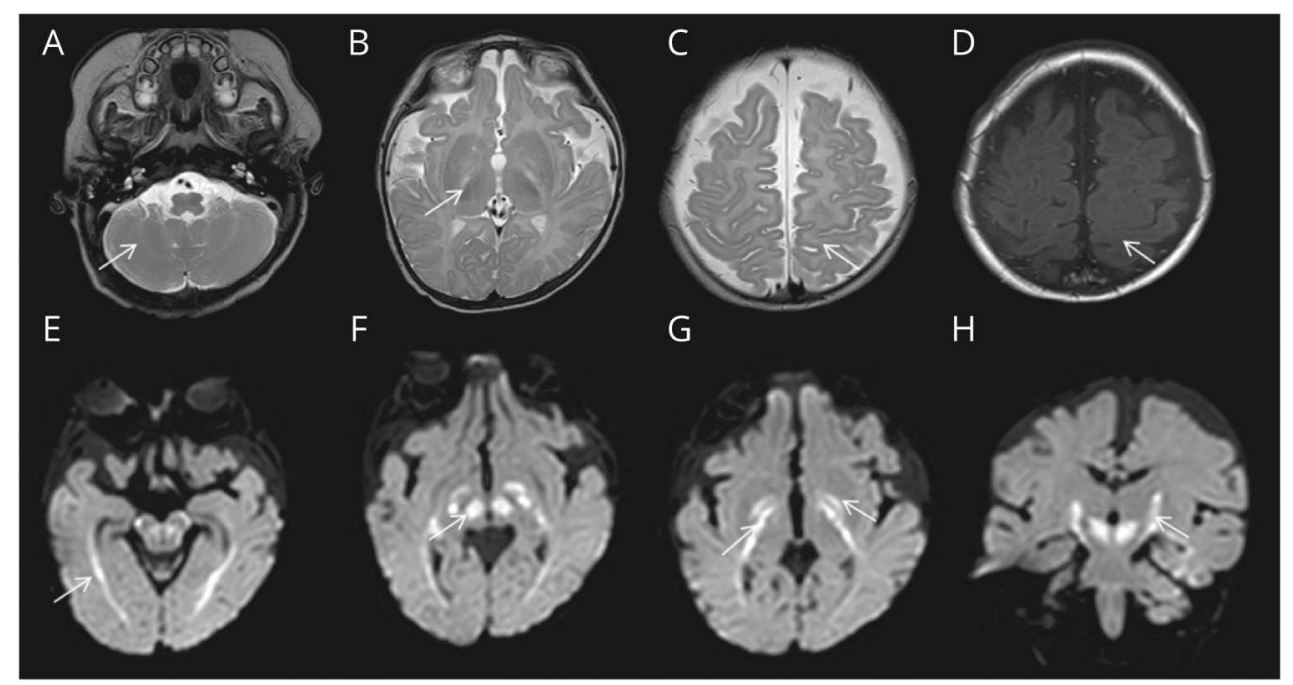

T2 (A-C) and T1-weighted (D) images depict lack of myelination in cerebellar white matter, posterior limb of internal capsule, and perirolandic regions (arrows). Diffusion-weighted imaging $(\mathrm{E}-\mathrm{H})$ shows diffusion restriction involving optic radiations $(E)$, red nucleus and cerebral peduncle region $(F)$, globus pallidus, and along corticospinal tract ( $\mathrm{G}$ and $\mathrm{H}$ ).

A 9-month-old girl presented with global developmental delay and refractory generalized seizures. Microcephaly, poor visual fixation, and intermittent dystonic posturing were observed on clinical examination. MRI brain (figure) revealed delayed myelination and restricted diffusion involving optic radiations, cerebral peduncles, red nuclei, globus pallidi, and corticospinal tract. EEG showed background slowing and multifocal epileptiform discharges. Workup revealed a homozygous, likely pathogenic variant in ITPA (c.124+1 G>A) and reduced inosine triphosphate pyrophosphohydrolase (ITPase) activity in skin fibroblasts $(0.19 \mathrm{nmol} / \mathrm{mg}$ protein $\times$ h, controls $6.86 \pm 2.51$ ). Imaging pattern of delayed myelination and restricted diffusion is suggestive of ITPase deficiency in a child presenting with early infantile epileptic encephalopathy. ${ }^{1,2}$

\section{Study Funding}

No targeted funding reported.

\section{Correspondence}

Dr. Morava

Morava-kozicz.Eva@

mayo.edu

\section{MORE ONLINE}

$\rightarrow$ Teaching slides

links.lww.com/WNL/

B334

From the Departments of Clinical Genomics (K.M., S.B., E.M.), Neurology, Pediatrics, Clinical Genomics (M.P.), and Laboratory Medicine and Pathology (E.M.), Mayo Clinic, Rochester, MN; Department of Laboratory Medicine (J.B.), Maastricht University, the Netherlands; University Children's Hospital (S.W.), Paracelsus Medical University (PMU), Salzburg, Austria; and Amalia Children's Hospital (S.W.), Radboud UMC, Nijmegen, the Netherlands.

Go to Neurology.org/N for full disclosures. Funding information and disclosures deemed relevant by the authors, if any, are provided at the end of the article. 


\section{Disclosure}

The authors report no disclosures relevant to the manuscript. Go to Neurology.org/N for full disclosures.

\begin{tabular}{lll}
\hline \multicolumn{2}{l}{ Appendix Authors } & \\
\hline Name & Location & Contribution \\
\hline $\begin{array}{l}\text { Karthik } \\
\text { Muthusamy, MD }\end{array}$ & $\begin{array}{l}\text { Clinical Genomics, Mayo } \\
\text { Clinic, Rochester, MN }\end{array}$ & $\begin{array}{l}\text { Designed and } \\
\text { conceptualized study, } \\
\text { analyzed the data, } \\
\text { drafted the manuscript } \\
\text { for intellectual content }\end{array}$ \\
\hline $\begin{array}{l}\text { Suzanne Boyer, } \\
\text { RDN, LD }\end{array}$ & $\begin{array}{ll}\text { Clinical Genomics, Mayo } \\
\text { Clinic, Rochester, MN }\end{array}$ & $\begin{array}{l}\text { Revised the manuscript } \\
\text { for intellectual content }\end{array}$ \\
\hline $\begin{array}{l}\text { Marc Patterson, } \\
\text { MD }\end{array}$ & $\begin{array}{l}\text { Neurology, Pediatrics and } \\
\text { Clinical Genomics, Mayo } \\
\text { Clinic, Rochester, MN }\end{array}$ & $\begin{array}{l}\text { Interpreted the data, } \\
\text { revised the manuscript } \\
\text { for intellectual content }\end{array}$ \\
\hline $\begin{array}{l}\text { Jorgen Bierau, } \\
\text { PhD }\end{array}$ & $\begin{array}{l}\text { Laboratory Medicine, } \\
\text { Maastricht University, the }\end{array}$ & $\begin{array}{l}\text { Interpreted the data, } \\
\text { revised the manuscript } \\
\text { for intellectual content }\end{array}$ \\
\hline
\end{tabular}

Appendix (continued)

\begin{tabular}{lll}
\hline Name & Location & Contribution \\
\hline $\begin{array}{l}\text { Saskia } \\
\text { Wortmann, } \\
\text { MD, PhD }\end{array}$ & $\begin{array}{l}\text { University Children's } \\
\text { Hospital, Paracelsus } \\
\text { Medical University } \\
\text { (PMU), Salzburg, }\end{array}$ & $\begin{array}{l}\text { Interpreted the data, } \\
\text { revised the manuscript } \\
\text { for intellectual content } \\
\text { Austria; Amalia } \\
\text { Children's Hospital, } \\
\text { Radboud UMC, }\end{array}$ \\
& $\begin{array}{l}\text { Nijmegen, the } \\
\text { Netherlands }\end{array}$ & \\
& Clinical Genomics and & Analyzed the data, \\
& Laboratory Medicine and & revised the manuscript \\
Pathology, Mayo Clinic, & for intellectual content \\
MD, PhD & Rochester, MN & \\
& &
\end{tabular}

\section{References}

1. Kevelam SH, Bierau J, Salvarinova R, et al. Recessive ITPA mutations cause an early infantile encephalopathy. Ann Neurol 2015;78:649-658.

2. Handley MT, Reddy K, Wills J, et al. ITPase deficiency causes a Martsolf-like syndrome with a lethal infantile dilated cardiomyopathy. PLOS Genet 2019;15: e1007605. 


\section{Neurology}

\section{Teaching NeuroImages: Neuroimaging Findings in Inosine Triphosphate Pyrophosphohydrolase Deficiency}

Karthik Muthusamy, Suzanne Boyer, Marc Patterson, et al.

Neurology 2021;97;e109-e110 Published Online before print February 16, 2021

DOI 10.1212/WNL.0000000000011719

\section{This information is current as of February 16, 2021}

\section{Updated Information \&} Services

References

Subspecialty Collections

Permissions \& Licensing

Reprints including high resolution figures, can be found at: http://n.neurology.org/content/97/1/e109.full

This article cites 2 articles, 0 of which you can access for free at: http://n.neurology.org/content/97/1/e109.full\#ref-list-1

This article, along with others on similar topics, appears in the following collection(s):

All Epilepsy/Seizures

http://n.neurology.org/cgi/collection/all_epilepsy_seizures All Genetics

http://n.neurology.org/cgi/collection/all_genetics

All Movement Disorders

http://n.neurology.org/cgi/collection/all_movement_disorders

Developmental disorders

http://n.neurology.org/cgi/collection/developmental_disorders

MRI

http://n.neurology.org/cgi/collection/mri

Information about reproducing this article in parts (figures,tables) or in its entirety can be found online at:

http://www.neurology.org/about/about_the_journal\#permissions

Information about ordering reprints can be found online:

http://n.neurology.org/subscribers/advertise

Neurology ${ }^{\circledR}$ is the official journal of the American Academy of Neurology. Published continuously since 1951, it is now a weekly with 48 issues per year. Copyright (C 2021 American Academy of Neurology. All rights reserved. Print ISSN: 0028-3878. Online ISSN: 1526-632X.

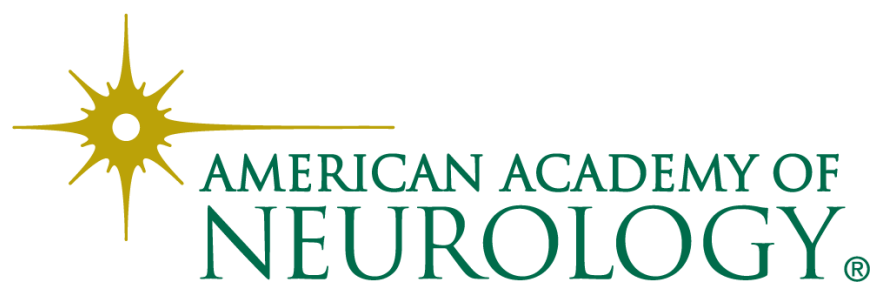

